\title{
La tecnología: un aliado en el aula
}

\author{
Technology: an ally in the classroom
}

\section{Mónica Lorena Pérez Torres Isabel Hernández Arteaga ${ }^{1}$}

\author{
Pérez T, Mónica. Hernández A, Isabel \\ miradas $\mathrm{N}^{\circ} 1-2018$ ISSN digital $\mathrm{N}^{\circ} 2539-3812$ Págs 162 - 176 \\ Recepción: Agosto 28 de 2017 \\ Aprobación: Mayo 13 de 2018 \\ Publicación: Junio 29 de 2018
}

\section{Resumen}

Este artículo de reflexión es el resultado de una investigación que permitió evidenciar las implicaciones de las tecnologías de la información y la comunicación (TIC) en la educación superior, particularmente en el proceso de formación profesional. Se demuestra la necesidad de que instituciones, docentes y estudiantes cambien el paradigma frente a su uso pedagógico, permitiendo que las TIC se conviertan en aliadas - y excusa perfectaque orienten el proceso de enseñanza-aprendizaje, para que los futuros profesionales estén en capacidad de participar efectivamente en la sociedad del conocimiento. De otra parte, se reconoce que su uso pedagógico posibilita al estudiante aprender mediante múltiples metodologías y desde diversos escenarios, geográficos y temporales, que facilitan el logro de las competencias profesionales que la sociedad y el mundo laboral demandan. Esta reflexión conlleva reconocer que el uso de las TIC en la formación profesional demanda la generación e innovación de estrategias didácticas que rompan las barreras espaciotemporales, exigiendo a los actores educativos una actualización crítica y permanente sobre la utilización de programas y dispositivos electrónicos como herramientas que apoyan el proceso de aprendizaje significativo.

1 Posdoctora en Investigación Cualitativa. Doctora en Ciencias de la Educación. Docente Investigadora Escuela de Posgrados UCC. Bogotá - Colombia. isabel.hernandez@ucc.edu.co 
Palabras clave: TIC, proceso de aprendizaje, formación profesional, práctica docente.

\section{Abstract}

This article of reflection is the result of a research that made it possible to demonstrate the implications of information and communication technologies (ICT) in higher education, particularly in the process of professional education. In this paper, likewise, it is proved the need for institutions, teachers, and students to have a shift in the pedagogical application, allowing understanding the ICTs as allies - and perfect excuse - to guide the teaching-learning process, to lead the skills of future professionals to participate effectively in the knowledge society. On the other hand, it is recognized that its pedagogical use enables the student to learn through multiple methodologies and from different geographical and temporal scenarios, which facilitate the achievement of the professional competencies that society and the labor world demand. This reflection entails recognizing that ICT use in vocational training, claims the creation and innovation of new didactic strategies able to break time-space barriers, claiming to the educative community to permanently and critically, update the software use and electronic devices employment as tools of knowledge acquisition.

Keywords: ICT, learning process, vocational training, teaching practice, higher education.

\section{Introducción}

Las tecnologías de la información y la comunicación (TIC) permiten que la sociedad evolucione de manera más rápida. $\mathrm{Al}$ mismo tiempo, facilitan el acto comunicativo, acortando distancias en el momento de entablar comunicación con los otros, y aportan a la sociedad, particularmente a la educación, puesto que se han convertido en aliadas didáctica en el quehacer de la práctica docente.

Cabe resaltar que en este artículo se entiende a las TIC como herramientas mediáticas mediadoras entre los protagonistas del escenario de formación profesional: el docente y el estudiante; además, se conciben como el instrumento que demanda - y a la vez permite — que se innove en la práctica docente, requiriendo que ella se encuentre a la vanguardia educativa, que su ejercicio realice desde la actualidad de los enfoques y tendencias en este campo, siendo capaz de hacer un uso pedagógico adecuado de los diferentes medios que los avances tecnológicos le ofrecen, abriendo la posibilidad de acceso al aprendizaje significativo. Sin embargo, factores como la escasez de competencias o la marcada reticencia de los docentes hacia el uso de las TIC en su quehacer académico han limitado su incursión en el ámbito pedagógico, circunstancia que va en detrimento del proceso de enseñanzaaprendizaje.

Este artículo tiene como objetivo reflexionar sobre las TIC en cuanto aliadas de los procesos de enseñanza-aprendizaje en la formación profesional. Esta reflexión parte del supuesto de que a partir de la segunda década del siglo XXI la tecnología transversaliza todo el sistema educativo, en calidad de herramienta mediadora del proceso de enseñanza-aprendizaje. La situación reviste aún más relevancia cuando se llega a la educación superior, toda vez que es allí donde se hace más 
necesaria la posesión de competencias en el uso de la tecnología.

Cabe reconocer que en el siglo XXI, las TIC permean casi todas las esferas de la vida humana, pues posibilitan la producción, el almacenamiento, el acceso, la presentación y la divulgación de un recurso absolutamente fundamental para la vida contemporánea: la información. Las TIC permiten el aprendizaje, la comunicación y el intercambio de opiniones; se caracterizan por disminuir distancias, haciendo el proceso de comunicación más eficiente. Por tal razón, se considera que si se dispone de la infraestructura tecnológica necesaria para cada uso, sus aplicaciones son casi ilimitadas.

Es preciso reflexionar sobre el hecho de que los sistemas educativos, en su desarrollo, tienden a cubrir las necesidades $\mathrm{y}$ demandas de la sociedad en los diferentes períodos histórico-culturales y, por tanto, de los modelos sociales. La vinculación a la cultura de los medios electrónicos, la digitalización y su influencia en todos los sectores de la sociedad, generan nuevos entornos de comunicación, que en la actualidad se sitúan en un espacio virtual. La gran influencia de las TIC y el enorme impacto que presentan en todos los órdenes sociales tiene que ver con el resultado de su implantación en los contextos sociales en los que se producen y con el uso que los ciudadanos hacen de las mismas, circunstancia que genera cambios en el soporte primordial del conocimiento, que transforman el modo de aprender, conocer y pensar de los seres humanos.

Para este artículo fue preciso revisar la teoría que existe a propósito del uso de las TIC en el ámbito pedagógico, entendidas como aliadas en el aula de clase. Esta búsqueda permitió reflexionar críticamente sobre su utilidad y las ventajas que proveen en la función que cumple la educación superior: formar profesionales competentes con capacidad de insertarse efectivamente tanto en la sociedad del conocimiento como en el mundo laboral.

Se asumen las TIC como herramientas mediadoras en el proceso de enseñanzaaprendizaje, es decir, entre el docente y el estudiante, sin límites en el tiempo y en el espacio geográfico. Con ello se posibilita que el cumplimiento del derecho básico del ser humano a la educación no esté restringido por el acceso, siendo necesario que el Estado provea la infraestructura necesaria para ello; asimismo, es menester que las instituciones de educación superior de país (IES) instalen y adecuen la infraestructura tecnológica requerida para que su comunidad académica, en su actuar educativo, sea parte activa de la sociedad del conocimiento.

El reto de la educación, como parte de un nuevo contexto social, es participar en la construcción de la sociedad del aprendizaje y del conocimiento a partir del uso de los recursos humanos y tecnológicos con los que cuenta la sociedad de la información. Ello implica cambios significativos en el rol del proceso de enseñanza-aprendizaje; requiere de recursos y de la formulación de políticas, programas, proyectos y acciones que fortalezcan la integración de las TIC al contexto educativo; demanda dinamizar el aprendizaje mediante la instrucción de docentes innovadores, formados en el uso pedagógico de las TIC, que procuren ambientes digitales para el aprendizaje de los estudiantes. 
Para ello es necesario insistir en que la educación ha cambiado a través de la historia, hecho que en la segunda década del siglo XXI se evidencia en las prácticas de los docentes y en el tipo e intereses de los estudiantes que se encuentran en las aulas de clase. Uno de los elementos al que se le atribuye dicha transformación son las TIC, porque ellas están involucradas en la evolución de todos los ámbitos de la sociedad.

\section{TIC y contexto educativo}

El estado del arte sobre el tema permite comprender la importancia, pertinencia y actualidad del asunto de interés de este artículo de reflexión. Son muchos los investigadores y académicos que han volcado su interés en esta relación y los cambios que comporta en el sistema social.

En primera instancia, conviene reflexionar sobre lo que Vygotsky (1988) señala a propósito del proceso de comprender las acciones realizadas por un individuo, en el que se hace necesario comprender las relaciones sociales en que ese individuo se desenvuelve, siendo necesario conocer el contexto social cotidiano donde el sujeto se apropia del conocimiento, determinado por su propia historia personal y social, es decir, por su interacción como sujeto social. Lo anterior provoca el aprendizaje tanto social como individual, que se interioriza en el individuo y que se explica en el proceso de internalización. Sobre el particular, Vygotsky (1987) advierte que todas las funciones superiores se originan como relaciones entre seres humanos, que dan sentido lógico al tratamiento del aprendizaje y el desarrollo. Es en este contexto donde adquiere sentido el papel de la educación y, particularmente, la relación docente-estudiante, pero también los contenidos, las estrategias metodológicas y los medios, relaciones en las cuales se accionan los aspectos comunicativos e interactivos que propicia el aprendizaje.

Por su parte, Pérez (2004) indica que el proceso de aprendizaje se origina en la relación del sujeto con el objeto, con los demás sujetos implicados en la situación de aprendizaje y consigo mismo, por tanto, el proceso de enseñanza-aprendizaje implica orientar el desarrollo del individuo como sujeto transformador de la realidad existente. Así entendido, el aprendizaje es una actividad social. La autora refiere que el foco de atención de Vygotsky es el sujeto activo, consciente, orientado hacia un objetivo; su interés se dirige hacia la interacción con otros sujetos y hacia sus acciones con el objeto, con la utilización de diversos medios, en condiciones sociohistóricas determinadas. El resultado fundamental del aprendizaje está constituido por las transformaciones endógenas y exógenas del sujeto, es decir, por los cambios psíquicos y físicos de quien aprende; mientras que las transformaciones en el objeto de la actividad sirven como medio para lograr las competencias requeridas.

De otro lado, los estudios de Tello (2008) permiten leer y reflexionar críticamente sobre el avance tecnológico. En primera instancia, el autor concibe el avance tecnológico como un progreso social discriminatorio porque no llega a todos los sectores sociales por igual, abriendo una brecha entre los que sí están integrados a esa nueva tecnología y los que no, marcando así grandes diferencias en el acceso, uso y beneficios de las TIC en los procesos educativos. Refiere también el autor que el concepto de sociedad de la información 
es un paradigma que produce grandes y profundas transformaciones, impulsadas principalmente por las nuevas herramientas para crear y divulgar información mediante tecnologías digitales, cuyos procesos se traducen en la creciente aparición de nuevas formas de organización social y productiva. Frente a esta realidad del campo educativo, particularmente, en la formación de profesionales, es necesario, en primera instancia, comprender el paradigma de la sociedad del conocimiento y sus implicaciones a nivel individual e institucional, con la finalidad de crear conciencia entre estudiantes y docentes sobre su responsabilidad social en el desempeño de roles.

A su vez, instituciones como la Unesco (2013) critican los modelos educativos diseñados para satisfacer las necesidades y retos de una sociedad diferente a la sociedad del conocimiento, y cuestiona qué es lo que se debe enseñar y cómo se debe aprender. En este contexto surgen grandes inquietudes sobre los actuales modelos pedagógicos y su utilidad para motivar el aprendizaje en la formación de profesionales, por ejemplo, ¿estos modelos educativos responden a los cambios culturales o son receptáculos de sus efectos?, ¿cómo está capitalizando la universidad las competencias de los estudiantes? En este horizonte, la universidad se enfrenta a la necesidad emprender transformaciones fundamentales que la trasladen, de una formación profesional para la sociedad industrial, a otra que prepara al estudiante para desempeñarse eficazmente en la sociedad del conocimiento. Y una de las competencias que requiere el profesional es el uso de las TIC, que en las aulas necesita una transformación de los roles de docentes y estudiantes.
Según Tello (2008), TIC es un término que en la actualidad agrupa a toda forma de tecnología usada para crear, almacenar, intercambiar y procesar información, en formas como datos, conversaciones de voz, imágenes (fijas o en movimiento), presentaciones multimedia y otras, incluyendo a aquéllas aún no concebidas. Las TIC se relacionan generalmente con el computador, el software y las telecomunicaciones; al mismo tiempo, tienen el objetivo de fortalecer y dar soporte a los procesos de operación y de negocios facilitando el tratamiento de cualquier tipo de información para incrementar la competitividad y productividad de las personas y organizaciones. De manera similar, Padilla, Vega y Rincón (2014) definen las TIC como el:

\section{(...) conjunto de elemen-} tos, recursos y estrategias coherentes con objetivos y fines de ordenar, manipular y difundir información en diversos campos y áreas del saber. Se sustentan en redes, terminales y protocolos que ofrecen servicios enmarcados en aspectos comunicativos e informáticos característicos de la actual sociedad de la información» (p. 281).

Las TIC, para Raudales (2014), son herramientas orientadas a contribuir al desarrollo de los países, cuya asimilación, apropiación e inclusión promueven la innovación, el crecimiento y la inclusión social. Las TIC son una importante fuente de generación de información y conocimiento $\mathrm{y}$, como tal, se constituyen en pilares fundamentales para el desarrollo económico y social, pues facilitan especialmente 
la prestación de servicios educativos mediante el desarrollo de plataformas que constituyen las bases y entornos virtuales del proceso de enseñanza-aprendizaje. En ese sentido, las TIC permiten la interacción docente-estudiante, ya sea de forma sincrónica o asincrónica, permitiendo resolver dificultades particulares de los estudiantes de manera más eficiente y amigable. Según el mismo Raudales, el campus virtual donde se desarrollan las TIC se describe como el lugar dedicado a la enseñanza, aprendizaje, transferencia de conocimientos e investigación, formado mediante la convergencia de múltiples aplicaciones de TIC: internet, web, comunicación electrónica, video, videoconferencia, multimedia y publicaciones electrónicas en general.

En el papel educativo de las TIC, Jiménez, Mora y Cuadros (2016) incluye los recursos utilizados con más frecuencia: la página web y el correo electrónico, y plataformas y aplicaciones, como Facebook, YouTube, Twitter, Windows Movie Maker, Kahoot y Audacity, destacando la competencia digital por lograr en los futuros profesionales. En tanto que para Contreras (2008), las TIC, como herramientas de aprendizaje, sirven en el proceso de aprendizaje desde la mera búsqueda de información hasta la realización de actividades en línea, vertiente que goza de mayor aplicación en la universidad, de acuerdo con las tres funciones básicas: información, comunicación y soporte didáctico. Según las tendencias pedagógicas, estas funciones vienen apoyando una metodología comunicativa, pero también existen escépticos que cuestionan la aplicación de las TIC en el campo de la educación. No obstante, a pesar de los reparos de algunos, resulta necesario entender que las
TIC llegaron a la escena educativa para quedarse y consolidarse.

Las TIC son consideradas por Gómez y Macedo (2010) como la innovación educativa del momento, que demanda de docentes y estudiantes cambios urgentes en el quehacer del proceso de formación profesional. Para los autores, las TIC permiten un fácil acceso a la información necesaria para orientar el aprendizaje, y se adaptan con facilidad a nuevas estrategias didácticas que estimulan el desarrollo cognitivo, creativo y divertido, en las áreas tradicionales del currículo. A su vez, Claro (2010) coincide con los autores en que las TIC son herramientas que forman parte de los componentes curriculares, como recursos pedagógicos, pero se desmarca de ellos al respecto de la realidad de las TIC en el aula de clase. Para Claro, esta innovación educativa pasa desapercibida para un buen número de docentes universitarios, quienes continúan con su rol transmisionista, considerándose aún, en esta época, los dueños del saber en sus disciplinas, y son temerosos de perder su estatus por cuenta de sus precarias competencias digitales.

Las TIC hacen parte del aula y, por tanto, del proceso de aprendizaje de los estudiantes. En la segunda década del siglo XXI, su uso parece no tener límites. Así lo manifiesta Dussel (2011), quien sugiere que las experiencias de los modelos educativos que usan intensivamente computadora, tablet, laboratorios de informática móviles, convivencia permanente con el celular y otros artefactos digitales con conexión a internet, muestran que las TIC llegaron para quedarse en el campo de la formación profesional, configurando una circunstancia que para Moro y Massa (2016) implica nuevas concepciones del proceso de 
aprendizaje, que conlleva nuevos roles $\mathrm{y}$ responsabilidades para el estudiante, transformándolo en participante activo y constructor de su propio aprendizaje, variando su interacción con el docente $\mathrm{y}$ creando ambientes interactivos de comunicación. Así pues, en el contexto de la sociedad del conocimiento, los actores del proceso educativo tienen la necesidad de adquirir competencias para el manejo de las TIC, consideradas transversales tanto en su formación profesional como en su posterior desempeño laboral.

En este sentido, se llama la atención de las IES para que pongan a disposición de sus estudiantes y docentes la infraestructura necesaria para el uso pedagógico de las TIC. Si bien existen buenas intenciones al respecto de profundizar el uso de las TIC como mediadoras del proceso de enseñanza-aprendizaje en aras de cimentar una formación profesional de calidad, sin la infraestructura tecnológica adecuada no es posible realizar ese propósito.

Es preciso puntualizar que en el aula, las TIC deben estar soportadas por varios elementos, entre ellos, el hardware, que según Ugalde (2004), es:

(...) una estructura dura, porque se refiere a aquellos elementos que el usuario no puede cambiar fácilmente. En términos prácticos, son todas las partes visibles o componentes de la computadora que facilita (sic) el almacenamiento e ingreso de información, entre otros, que se clasifican en dispositivos de almacenamiento, de entrada y de salida (p. 67).
En contraste, según el mismo Ugalde (2004), el software es una:

(...) estructura suave, es decir, que puede ser modificada por el usuario a partir de la manipulación de los programas que contiene; en español se traduce como -programa- y se aplica a toda colección de instrucciones que sirven para que la computadora ejecute y realice tareas (p. 11).

La actualización permanente de estos dos elementos es indispensable para el uso adecuado y eficiente de las TIC, como aliadas en el proceso de formación profesional.

En este orden de ideas, el hardware y el software son elementos indispensables para la informática del siglo XXI, pues son los soportes técnico-tecnológicos que posibilitan la ejecución de tareas en un entorno TIC y son el sustrato de las aplicaciones y plataformas digitales. De acuerdo con Mora (2002), se entiende por aplicación web a un tipo especial de aplicación cliente/servidor, donde tanto el cliente (el navegador, explorador o visualizador) como el servidor (servidor web) y el protocolo mediante el que se comunican (HTTP), se encuentran estandarizados y no han de ser creados por el programador de aplicaciones, es decir, que ella ya está constituida y que el usuario solo debe utilizarla con la configuración predeterminada. Es por ello que estudiantes $\mathrm{y}$ docentes deben adquirir las competencias necesarias para su uso eficiente en el campo de la formación profesional. 
$\mathrm{Al}$ respecto de los fondos destinados en las últimas dos décadas por el sector educativo para ponerse al día en las últimas TIC, IIPE-Unesco (2006) subrayan que las IES han realizado significativas inversiones en hardware y en infraestructura informática, lo que potencia la importancia del software educativo (herramientas y aplicaciones) y de los contenidos digitales (materiales de aprendizaje). Sin embargo, advierten que las IES han destinado el mayor porcentaje de esas inversiones a la adquisición de hardware y conexiones, dejando un estrecho margen para la inversión en software y contenidos educativos. Si bien la tendencia es que esta proporción se equilibre en el futuro, hoy es generalizado el lamento de los profesores sobre la falta de materiales TIC adecuados para la enseñanza y la orientación del aprendizaje de sus estudiantes.

En el pasado, el aprendizaje del manejo de estas aplicaciones ocupó un espacio importante en los currículos, pero en la actualidad ha disminuido por cuanto los estudiantes llegan a la universidad sabiendo usar las herramientas tecnológicas. De allí que los currículos prioricen ahora el aprendizaje del manejo de aplicaciones específicas para la vida profesional.

De igual manera, existen plataformas educativas gratuitas que ayudan a los docentes con la creación de páginas de cátedra y cursos en línea, que también dan acceso libre a los estudiantes. El uso de estas herramientas es cada vez más frecuente entre docentes y estudiantes con deseos de incorporar las TIC en su proceso de enseñanza-aprendizaje. En el siglo XXI, las aplicaciones de currículum en línea son una estrategia didáctica que permite la digitalización de documentos y su puesta en línea. Ampliamente utilizada en el mundo, es una estrategia a la que acuden aquellos países que presentan excelentes resultados en sus procesos formativos. El currículum en línea es una propuesta didáctica que permite profundizar el aprendizaje de temas académicos y que posibilita además el uso de actividades para avanzar al ritmo de los estudiantes.

Para Muraro (2005), el aprendizaje asistido por computador es generalmente ofrecido por las IES mediante plataformas orientadas a ofertar formación profesional en la modalidad virtual. Estas plataformas son diseñadas para que los estudiantes trabajen individualmente en busca de su aprendizaje, pero soportan la interacción docente-estudiante y estudiante-estudiante. Esta modalidad de formación exige la orientación y el acompañamiento de personal con competencia y experiencia en estos ambientes de aprendizaje. Para acceder a estos espacios emergentes, los docentes universitarios deben saltar el linde que los separa del uso de las tecnologías y volverlas sus aliadas en el proceso de formar profesionales competentes.

En este contexto, es preciso referirse a los objetos virtuales de aprendizaje (OVA), como un conjunto de recursos digitales, autocontenibles y reutilizables, que tienen como propósito impactar el aprendizaje de los estudiantes. Los OVA están constituidos por tres componentes internos: contenidos, actividades de aprendizaje y elementos de contextualización. El objeto de aprendizaje debe presentar una estructura de información externa (metadatos), la cual facilita su almacenamiento, identificación y recuperación. Payares (2014) define el OVA como una herramienta de aprendizaje que señala a los sujetos dentro de los 
procesos de agenciamiento y que brinda conceptos que abren brechas en los cambios y transformaciones que exige la sociedad moderna. A propósito, Boshell (2008) añade que el diseño de los OVA debe cumplir con los aspectos propios de la disciplina objeto de aprendizaje y con las metodologías para la creación de materiales educativos y usos de la tecnología que miden el aprendizaje. En la actualidad, los OVA cumplen un papel activo en la formación de profesionales, por lo que el docente debe ser experto en su diseño, manejo, implementación y uso pedagógico, tanto en el proceso de enseñanza como en la orientación del proceso de aprendizaje del estudiante.

Crear ambientes o aulas propicias para el aprendizaje es una función docente. Así pues, los ambientes virtuales de aprendizaje (AVA) corresponden, según Rico (2011), a un conjunto de elementos que relacionan la comunicación y la pedagogía, dispuestos en el sistema de gestión de aprendizaje (LMS), que permiten acceder y canalizar las diferentes estrategias y actividades didácticas en busca de una transformación en la estructura cognitiva del estudiante y asegurar la eficacia en el proceso de aprendizaje significativo. Las TIC, plantea Rico, «constituyen un apoyo excelente en el aprendizaje (...), ya que permiten observar, escuchar, dinamizar, manipular e interactuar con los fenómenos reales, simulados en ambientes virtuales, motivando el interés del estudiante» (p. 7) por el conocimiento; además, continúa el autor, los AVA «se basan en el principio de aprendizaje colaborativo donde se permite a los estudiantes realizar sus aportes» (p. 15); asimismo, se apoyan en herramientas multimediales que hacen más agradable el aprendizaje, dejando de ser simplemente un texto en línea para transformarse en un entorno interactivo de construcción de conocimiento. Los AVA motivan y fortalecen el aprendizaje autónomo en los estudiantes, ya que a través de internet tienen permanente acceso a toda la información y a las aplicaciones interactivas (Rico, 2011, p. 6).

Sobre el papel que cumplen los AVA en el proceso formativo, Ausubel, Novak y Hanesian (2000) manifiestan que sacan al estudiante de la educación cerrada y lineal y lo impulsan a participar en escenarios como foros de debate, comentarios y aplicaciones interactivas donde puede expresar sus ideas de manera espontánea y sin límite de tiempo o presión del docente; no obstante, usarlos requiere de unas competencias básicas sobre el manejo de las TIC.

La realidad es que las TIC, hoy por hoy, revisten una influencia cada vez mayor en la forma como nos comunicamos y relacionamos los seres humanos, entre nosotros mismos y con nuestros contextos, hecho que transforma nuestras maneras de aprender, de vincularnos con los demás y de desempeñarnos en sociedad. Al respecto, la reflexión tiene que ver con el reto que comporta hacer un uso eficaz de las TIC para que estén al servicio de los intereses de los estudiantes, los docentes y, en general, de toda la comunidad educativa para que impacten su función fundamental: la formación pertinente y de calidad de profesionales.

Las competencias en TIC desarrolladas en el contexto universitario posibilitan procesos de formación de calidad cuando contribuyen al desarrollo profesional de los docentes y a la mejora de la gestión y 
la administración de la educación superior. Pero ello está sujeto a la formulación de políticas tendientes a desarrollar y consolidar estas capacidades en las IES, si se quiere que con el uso de las TIC se generen proceso procesos educativos con sentido humano.

\section{Las TIC, aliadas del aprendizaje}

La educación debe ir a la par de los diferentes avances sociales y tecnológicos que se gestan a través del tiempo. La educación es un derecho y un proceso permanente que no puede apartarse del avance tecnológico. Una de las principales razones para que las TIC hayan evolucionado tanto y tan rápido en los últimos años es que la globalización las incluye en todos los campos del conocimiento y del quehacer humano, lo que obliga a la educación a aceptar que están inmersa en sus procesos y a que haga el mejor uso de las mismas. Así lo entiende la Unesco (2013) cuando afirma que la educación debe ser coherente con el momento histórico y tecnológico que se está viviendo. Esta aserción implica que la educación debe tomar consciencia de lo que significan las TIC para la sociedad gracias a que ellas no solamente son un conjunto de herramientas tecnológicas sino que tienen una intencionalidad, la cual se refleja en las nuevas formas de comunicación que se establecen cuando entablamos vínculos sociales y familiares, en el acceso a las distintas fuentes de información y al crear nuevas maneras de ver el mundo.

Adicionalmente, es necesario encontrarle sentido a la transformación educativa mediada por las TIC. Sobre este aspecto, se propone que para su utilización se debe considerar el propósito de uso e implementación en el proceso de aprendizaje, dado que benefician al estudiante en el logro de competencias e impactan el currículo. La experiencia del uso de las TIC en el proceso de aprendizaje se percibe de varias maneras, tal como afirman Sunkel, Trucco y Espejo (2014). Indica que se puede aprender haciendo uso de los diferentes medios que la tecnología provee, pero que, a su vez, se obtiene un aprendizaje sobre estas. Es por esto que las TIC son apreciadas como un objeto de enseñanza, perotambién como herramientas aplicables al proceso de aprendizaje. Por consiguiente, la reflexión en este punto es que los estudiantes deben tomar una posición, toda vez que ellos son el centro del proceso de formación profesional que requiere hacer uso adecuado de las herramientas existentes, adecuándolas a su quehacer como discentes.

Cabe resaltar que docentes y estudiantes son los principales actores del proceso educativo, por tanto, son imprescindibles para la incorporación de las TIC al aula de clase. Según afirman Riascos, Quintero y Ávila (2009), la percepción de estos dos actores es innegable en la inmersión de las TIC en las IES, donde considera tres variables: percepción de uso, grado de utilización e impacto. Para las autoras, considerar estas variables permite optimizar los procesos educativos que se dan en el acto pedagógico; aunque advierte, también, que no es suficiente que docentes $\mathrm{y}$ estudiantes tengan concepciones $\mathrm{y}$ percepciones positivas frente al uso de las TIC, ya que se hace necesario contar con los recursos y la infraestructura tecnológica y con las competencias para su manejo, tanto por el cuerpo docente como por los estudiantes.

Sobre estas competencias genéricas, Riascos, Quintero y Ávila (2009) señalan 
que los estándares deben estar relacionados con cinco competencias: 1) poseer el conocimiento teórico y práctico sobre el uso de las TIC en los procesos educativos, tanto por quien orienta el aprendizaje como por quien opta por ese aprendizaje; 2) planear, diseñar y participar en los AVA; 3) comprender y diseñar el currículo teniendo en cuenta las TIC en su desarrollo, sin olvidar que los estudiantes son el centro del proceso; 4) valorar y evaluar el logro de competencias haciendo uso de diferentes técnicas para verificar el proceso; 5) productividad y práctica profesional, que corresponde a la autoevaluación docente sobre su quehacer e impacto profesional.

Por su parte, Galvis (2000) propone que los usos de las TIC en el proceso de aprendizaje se clasifican en función de tres objetivos, los cuales se establecen de acuerdo con los ambientes de aprendizaje: 1) apoyar la transmisión de mensajes al estudiante por medio de tutoriales, ejercitadores y sitios web informativos; 2) apoyar el aprendizaje activo mediante la experimentación con los objetos de estudio a través de simuladores de procesos, calculadoras, juegos de actividad, competencias o roles, paquetes de procesamiento estadístico de datos, navegadores y herramientas de productividad; 3) facilitar la interacción mediante juegos en red colaborativos, mensajería electrónica, correo electrónico, foros, video $\mathrm{o}$ audioconferencia. $\mathrm{Al}$ respecto, es posible afirmar críticamente que la mayoría de los estudiantes son expertos en el uso social de estas herramientas, pero también que no las valoran como un recurso indiscutible para su proceso de formación; entonces, vendría bien una toma de conciencia por parte de los estudiantes sobre la utilidad pedagógica de las TIC.
En la misma línea, Jaramillo, Castañeda y Pimienta (2009) vinculan la propuesta de clasificación de usos de las TIC en el aprendizaje con cuatro categorías: 1) enseñar; 2) practicar y ejercitar; 3) simular, resolver problemas y elaborar productos; 4) proveer acceso a la información y servir como medio de comunicación con otras personas. Se recoge también la clasificación que Hopper y Rieber (1995) proponen acerca de la relación docenteestudiante, compuesta por cinco niveles: 1) familiarización sobre el uso de las TIC en actividades personales: el docente aprende a usar el computador y algunos programas, pero no lleva esos aprendizajes al campo educativo; 2), utilización: el docente lleva las TIC al aula, pero no lo hace con un propósito pedagógico sino para facilitar su labor administrativa; 3) integración consciente: el docente decide delegar algunas tareas y operaciones a las TIC para apoyar su labor; 4) reorientación: el docente asume el rol de facilitador de la construcción de conocimientos en el ambiente de aprendizaje, destacando que el foco del proceso es el estudiante; 5) evolución: el docente mejora la práctica pedagógica con base en sus nuevos conocimientos y en cómo aprenden las personas con las nuevas herramientas tecnológicas. Los dos últimos niveles sitúan al docente en la misión que debe cumplir cuando apoya la formación de profesionales y lo ubican como orientador del estudiante en la construcción de su propio conocimiento a partir del reconocimiento de la evolución de la práctica pedagógica que comporta las TIC.

Llegados a este punto, resulta pertinente recordar la observación que Lugo (2010) hace al respecto de las reformas que involucró el arribo de las TIC a la 
universidad, cuando advierte que gracias a las TIC el estudiante adquiere mayor nivel de autonomía y responsabilidad en el proceso de su propio aprendizaje, obligando al docente a salir de su clásico rol como única fuente de conocimiento y demandando de la universidad respuestas frente a los retos que los nuevos paradigmas y tendencias de la formación de profesionales imponen.

Las TIC, como aliadas del docente y del estudiante en el aula, han de permitir incrementar el logro de competencias, tanto del docente en los procesos de enseñanza como del estudiante en sus procesos de aprendizaje, para lo cual se requiere la implementación consciente de políticas para su utilización en la formación de profesionales. Para ello, las metodologías virtuales son oportunas porque llaman la atención del docente universitario y lo impulsan a que asuma un cambio de paradigma, para posicionarse en las tendencias educativas y tecnológicas que le garanticen el logro de competencias y su implementación pedagógica, con el propósito de orientar la construcción del conocimiento por parte del estudiante. $\mathrm{Al}$ mismo tiempo, es necesario orientar el uso y desarrollo de programas y recursos educativos que promuevan las TIC para una educación inclusiva, proporcionando espacios de asesoría que expandan la cobertura de las TIC sobre todo el sistema educativo, en general, y al aula, en particular, para que esa comunidad se beneficie de todo el potencial que ellas tienen.

\section{Consideraciones finales}

En conclusión, es importante resaltar que la educación universitaria debe estar dispuesta a incluir las TIC en la práctica diaria de los procesos de enseñanza-aprendizaje. Si lo consigue, la universidad demostrará que está en sintonía con el momento histórico y tecnológico que está viviendo; pero, si no, sencillamente la IES quedarán rezagada de las tendencias educativas, y los estudiantes sin las competencias tecnológicas que exige la vinculación efectiva a la sociedad y el mundo laboral contemporáneos. Por consiguiente, es fundamental que el profesional del siglo XXI haga un uso pedagógico consciente y responsable de las TIC, ya que su vinculación a diferentes modalidades de formación profesional le exigirán la posesión de competencias para la utilización e innovación de programas, tanto en el manejo teórico como en los procesos prácticos de su profesión.

La universidad tiene una responsabilidad social que debe cumplir: disminuir la brecha que existe entre quienes tienen acceso a las TIC y quienes no lo tiene, ya que las TIC están inmersas en la vida cotidiana de las personas. Entonces, desde el proceso de formación profesional, la universidad debe posibilitar la culturización tecnológica de los estudiantes en cuanto al uso pedagógico de las TIC, con miras a su aplicación en su campo profesional de desempeño. En ese sentido, la universidad facilita la renovación de los modelos de enseñanza-aprendizaje, del material utilizado para este proceso; así, se motiva el logro de competencias tecnológicas específicas de la profesión. Para cumplir esta responsabilidad, la universidad debe proveer a docentes y estudiantes la infraestructura tecnológica de redes, equipos y software que permitan su uso pedagógico eficiente; además de la capacitación necesaria al estamento docente encargado de orientar su utilización pedagógica para la mejora de la calidad de la formación profesional. 
El aprendizaje cooperativo y solidario se ha desarrollado en un contexto educativo de caráctertradicional, esdecir, enlainteracción profesor-estudiante bajo la modalidad presencial. Este tipo de aprendizaje hace un giro hacia un vértice que le asigna un nuevo sentido y significado a la formación cuando se transpone a un ambiente virtual, que abre la puerta a la posibilidad de cooperar y favorece la capacidad de compartir información y conocimiento. El aprendizaje colaborativo y solidario se hace más visible en ambientes virtuales cuanto más apartados se encuentran física o geográficamente docente y estudiante; cuando los protagonistas del proceso se alejan de las formas tradicionales de enseñar y aprender en un ambiente presencial y se vinculan a contextos dinámicos, donde la presencialidad no es significativa, se incrementa el nivel de autonomía frente al proceso mismo de aprendizaje.

Una vez los docentes permitan la entrada de las TIC, en cuanto recurso aliado, a su práctica pedagógica, podrán evaluar objetivamente los resultados de la utilización de las mismas. Este ejercicio transforma de raíz la visión retrógrada de su uso porque dejan de ser el contenido de una asignatura más, para convertirse en una herramienta indispensable en el quehacer del docente universitario; a partir de ellas, el docente puede crear e innovar sus prácticas pedagógicas y romper las barreras geográficas y temporales de acceso a la formación profesional. Esta reflexión permite comprender la transformación que se ha gestado en la manera de pensar y comprender los actos de enseñar y aprender, donde el rol del docente es de orientador del proceso de creación del conocimiento por parte del estudiante, quien adquiere un rol activo y principal en su aprendizaje significativo.

Las revoluciones tecnológicas -en particular, la de las TIC - han provocado modificaciones tan grandes en la estructura de la vida social, que han conducido a transformar la identidad cultural de los pueblos. El uso pedagógico eficiente de las TIC en la formación de profesionales permite un aprendizaje autónomo, colaborativo, solidario y significativo, que transciende lo repetitivo y lineal, para dar cabida a lo complejo y dinámico. Es por ello que la universidad está llamada a moldear la identidad de los estudiantes para que puedan asignar significados a su existencia y logren dinamizar su quehacer en sus contextos, tanto en el inmediato como en los más amplios. La universidad tiene la obligación de repensar las competencias del ser, del conocer, del hacer y del convivir con las que forma a sus profesionales, para que sean capaces de reflexionar críticamente sobre el mundo en el que desplegarán los conocimientos adquiridos en la institución. Solo así serán capaces de evaluar si el contexto educativo que los formó es el más propicio para aprender, crear, innovar, desarrollar y poner al servicio del ser humano los conocimientos necesarios para crear un mundo con calidad de vida.

El avance de las TIC en el ámbito educativo -expresado como un medio de aprendizaje - posibilita la mejora del proceso de enseñanza y de creación y consolidación de conocimientos; también auspicia la formulación de propuestas de solución viables a problemas sociales reales, la toma de decisiones pertinentes, y el sentido de pertenencia y responsabilidad con el medio ambiente. Asimismo, el proceso de enseñanza-aprendizaje 
mediado por las TIC dinamiza el proceso de crear, innovar y asimilar la tecnología. Lo anterior es posible solamente si, en este caso, las instituciones de educación superior invierten y fortalecen los procesos tecnológicos desde los cuales contribuyen al desarrollo de las regiones.

\section{Referencias}

Ausubel, D., Novak, J. y Hanesian, H. (2000). Psicología educativa un punto de vista cognoscitivo. $13^{\text {ed }}$. México: Trillas.

Boshell, G. (2008). Objetos virtuales de aprendizaje con herramientas de Internet2 OVAs-2. (Tesis de Doctorado en Educación). Bogotá: Universidad Santo Tomás.

Claro, M. (2010). Impacto de las TIC en los aprendizajes de los estudiantes. Estado del arte. Colección Documentos de Proyectos No. 339. Santiago de Chile: CEPAL.

Contreras, N. (2008). La enseñanza-aprendizaje de lenguas extranjeras y las TICs: el caso del español como lengua extranjera (ELE). Iniciación a la Investigación e3, a4, pp. 1-7. Disponible en: https://revistaselectronicas. ujaen.es/index.php/ininv/article/view/233

Dussel, I. (2011). Aprender y enseñar en la cultura digital documento básico. VII Foro Latinoamericano de Educación. Experiencias y aplicaciones en el aula. Aprender y enseñar con nuevas tecnologías. Buenos Aires: Fundación Santillana.

Galvis. (2000). Propuesta de los usos de las TIC para aprender y enseñar. Bogotá: Macmillan.

Gómez, L. y Macedo, J. (2010). Importancia de las TIC en la en la educación básica regular. Investigación Educativa, 14(25), pp. 209-224. Disponible en: https://educrea.cl/importancia- de-las-tic-en-la-educacion-basica-regular/

Hooper, S. y Rieber, L. (1995). Teaching with technology. En Ornestein (Edit.). Teaching: Theory into practice. (pp. 154 - 170) Needham Heights, MA: Allyn and Bacon.

IIPE-UNESCO. (2006). La integración de las TecnologíasdelaInformaciónyla Comunicación en los Sistemas Educativos. Estado del arte y orientaciones estratégicas para la definición de políticas educativas en el sector. Buenos Aires: Unesco. Disponible en: http://unesdoc.unesco. org/images/0015/001507/150785s.pdf

Jaramillo, P., Castañeda, P. y Pimienta, M. (2009). Qué hacer con la tecnología en el aula: inventario de usos de las TIC para aprender y enseñar. Educación y Educadores, 12(2), pp. 159-179. Disponible en: http:// educacionyeducadores.unisabana.edu.co/ index.php/eye/article/view/1492/1661

Jiménez, D., Mora, M. y Cuadros, R. (2016). La importancia de las nuevas tecnologías en el proceso educativo. Propuesta didáctica TIC para ELE: mELEndien7dias. Revista Fuentes, 18(2), pp. 209-223. Disponible en: https://ojs. publius.us.es/ojs/index.php/fuentes/article/ view/2814/2743

Lugo, M. (2010). Las políticas TIC en la educación de América Latina. Tendencias y experiencias. Revista Fuentes, 10, pp. 52-68. Disponible en: https://ojs.publius.us.es/ojs/ index.php/fuentes/article/view/2587/2415

Mora, S. (2002). Programación de aplicaciones WEB: Historia, principios básicos y clientes Web. Alicante: Universidad de Alicante.

Moro, L. y Massa, S. (2016). Aprendizaje de ciencias naturales mediado con TIC: estudio de caso de una experiencia innovadora. Disponible en: http://reposital.cuaed.unam.mx:8080/jspui/ 
bitstream/123456789/4828/1/VE16.622.pdf

Muraro, S. (2005). Una introducción a la informática en el aula. Buenos Aires: Fondo de Cultura Económica.

Padilla B, J., Vega, P. y Rincón, D. (2014). Tendencias y dificultades para el uso de las TIC en educación superior. Entramado, 10(1), enejul, pp. 272- 295. Disponible en: http://www. scielo.org.co/pdf/entra/v10n1/v10n1a17.pdf

Payares, J. (2014). Relación que se establece entre los objetos virtuales de aprendizaje (OVAS) y las prácticas pedagógicas de los docentes del Bachillerato virtual de la universidad Gran Colombia. (Tesis de Maestría en Desarrollo Educativo y Social). Bogotá: Universidad Pedagógica Nacional. Disponible en: http://repositorio.pedagogica.edu.co/xmlui/ bitstream/handle/123456789/899/TO-17311. pdf?sequence $=1$

Pérez, M. (2004). Freire y Vygotski en los procesos educativos latinoamericanos contemporáneos. Recuperado de: http:// www.cubaarqueologica.org/document/ant06 perezperez.pdf

Raudales, N. (2014). El uso de las tecnologías de información como medio de alfabetización en las instituciones de educación de Honduras. Revista Economía y Administración E\&A, 5(1), pp. 79-98. Disponible en: http://www. iies-unah.org/Revista/index.php/EyA/article/ view/121

Riascos, S., Quintero, D. y Ávila, G. (2009). Las TIC en el aula: percepciones de los profesores universitarios. Educación y Educadores, 12(3), pp. 133-157. Disponible en: http://www. redalyc.org/articulo.oa? $\mathrm{id}=83412235008$

Rico, C. (2011). Diseño y aplicación de ambiente virtual de aprendizaje en el proceso de enseñanza-aprendizaje de la física en el grado décimo de la IE. Alfonso López Pumarejo de la ciudad de Palmira. (Tesis de Maestría en Enseñanza de las Ciencias Exactas y Naturales). Palmira: Universidad Nacional de Colombia. Disponible en: http://www.bdigital. unal.edu.co/5737/1/7810039.2011.pdf

Sunkel, G., Trucco, D. y Espejo, A. (2014). La integración de las tecnologías digitales en las escuelas de América Latina y el Caribe. Una mirada multidimensional. Libros de la CEPAL, No. 124 (LC/G. 2607-P). Santiago de Chile: CEPAL. Disponible en: http://repositorio.cepal. org/handle/11362/36739

Tello, E. (2008). Las tecnologías de la información y comunicaciones (TIC) y la brecha digital: su impacto en la sociedad de México. Revista de Universidad y Sociedad del conocimiento, 4 (2), pp. 1-8. Disponible en: http://www.uoc.edu/rusc/4/2/dt/esp/tello.pdf

Ugalde, M. (2004). Nuevas Tecnologías, Procedimientos básicos e ideas de aplicación en la educación especial. San José costa Rica: Editorial Universidad Estatal a Distancia (UNED).

Unesco. (2013). Enfoques estratégicos sobre las TICS en educación en América Latina y el Caribe. Santiago de Chile: Unesco. Disponible en: http://www.unesco.org/new/fileadmin/ MULTIMEDIA/FIELD/Santiago/images/ ticsesp.pdf

Vygotsky, L. S. (1987). Historia del desarrollo de las funciones psíquicas superiores. La Habana: Editorial Científico-Técnica.

Vygotsky, L. (1988). Pensamiento y lenguaje. México: Ediciones Quinto Sol. 